\title{
Optical Interferometers with Reduced Sensitivity to Thermal Noise
}

\author{
H. J. Kimble, ${ }^{*}$ Benjamin L. Lev, ${ }^{\dagger}$ and Jun Ye \\ JILA, National Institute of Standards and Technology and University of Colorado, Boulder, Colorado 80309-0440, USA
}

(Received 20 June 2008; published 30 December 2008)

\begin{abstract}
A fundamental limit to the sensitivity of optical interferometry is thermal noise that drives fluctuations in the positions of the surfaces of the interferometer's mirrors, and thereby in the phase of the intracavity field. Schemes for reducing this thermally driven phase noise are presented that rely upon the coherent character of the underlying displacements and strains. Although the position of the physical surface fluctuates, the optical phase upon reflection can have reduced sensitivity to this motion. While practical implementation of such schemes for coherent compensation face certain challenges, we hope to stimulate further work on this important thermal noise problem.
\end{abstract}

DOI: 10.1103/PhysRevLett.101.260602

Thermal noise presents a fundamental limit to measurement sensitivity in diverse areas of science and technology [1]. One important setting is that of optical interferometry in which otherwise stable structures experience small, thermally driven fluctuations in their dimensions in applications ranging from frequency metrology [2,3], to gravitational wave detection [4,5], to the realization of quantum behavior for macroscopic objects [6].

In some cases, the dominant limitation to length stability originates from thermally driven displacement noise for the reflective surfaces of the mirror substrates, and not from the supporting structure $[2,3,5]$. These fundamental fluctuations arise from substrate dissipation as demanded by the fluctuation-dissipation theorem (FDT) [7-9].

Beyond displacements driven by thermal noise in the substrate itself [10-15], diverse other sources of mechanical noise have been identified, including frictional losses in the materials that form the mirror coating $[13,16]$, thermoelastic damping in the substrate and coating $[15,17,18]$, and thermorefractive noise [19]. Measurements in rigid [2,3] and suspended interferometers [20-22] have confirmed many characteristics of such noise sources.

Various avenues have been followed for reducing thermal noise in optical interferometers, the most significant being lowering mechanical losses for the substrate [1,23] and, more recently, the coating $[22,24]$. New designs for advanced interferometers include the use of "coating-free mirrors" [25,26] and short Fabry-Perot (FP) cavities [27].

In this Letter, we propose a new strategy for reducing thermally driven phase noise in optical interferometers. Fundamental to our proposal is the observation that various manifestations of thermal noise can be coherent one with another. Our examples recognize that stochastic displacements $\delta u_{z}$ perpendicular to the mirror's surface are necessarily accompanied by correlated strains and index changes in the materials of the mirror coating and substrate. Normally, the phase shift $\delta \beta$ due to these strains are small compared to the shift $\delta \theta$ from the surface motion $\delta u_{z}$. However, by suitable design, it is possible to achieve a total
PACS numbers: 07.60.Ly, 04.80.Nn, 05.40.Ca, 42.62.Eh

phase shift $\delta \Phi=\delta \theta+\delta \beta \ll \delta \theta$ for the reflected field. That is, although the physical surface of the mirror is subject to random displacements $\delta u_{z}, \delta \Phi$ can have reduced sensitivity to these displacements with $\delta \theta$ coherently compensated by $\delta \beta$ from the coating and substrate. Although our current analysis is for "Brownian noise" of the substrate, our methodology should also be applicable to thermoelastic and coating noise as well.

As a first example, consider thermal noise for an eigenmode of a cylindrical mirror with mechanical resonance frequency $\omega_{0}$. For frequencies $\omega \simeq \omega_{0}$ and quality factor $Q_{0} \gg 1$, microscopic thermal noise excites the entire "shape" of the relevant eigenmode $\boldsymbol{\xi}_{0}(\boldsymbol{r})$, with a single, overall amplitude set by equipartition of energy $[10,11]$. The amplitude $\delta u_{z}$ and accompanying strain $\epsilon_{z z}$ for thermally driven motion perpendicular to the mirror surface at $z=0$ are then determined directly from $\boldsymbol{\xi}_{0}(\boldsymbol{r})$.

Figure 1(a) illustrates a particular axisymmetric eigenmode $\boldsymbol{\xi}_{0}(\boldsymbol{r})$ for a substrate of mass $M$ as determined from a numerical finite-element analysis [28]. The end faces at $z=0, l$ oscillate in opposition about the plane at $z=l / 2$ with frequency $\omega_{0}$. At temperature $T, \delta u_{z}$ at the central points $x, y=0$ on the end faces have amplitude $\left\langle\delta u_{z}^{2}\right\rangle_{\omega_{0}} \simeq$ $k_{B} T / M_{0} \omega_{0}^{2}$, where $M_{0}=2.7 M$ is the effective mass for $\boldsymbol{\xi}_{0}(\boldsymbol{r})$ [11]. Significantly, the axial strain per unit displacement $\zeta \equiv \epsilon_{z z} / u_{z} \simeq-1600 / \mathrm{m}$ at $\boldsymbol{r}=0$ in Fig. 1(a).

Light reflected from the fluctuating surface of the substrate will be phase shifted. Following Levin [13], we introduce the displacement $q(z)=\int d x d y \delta u_{z}(r, z) \psi(r)$ weighted by the normalized intensity $\psi(r)$ over a plane at depth $z \geq 0$, where $\psi(r)=\left(2 / \pi w_{0}^{2}\right) \exp \left(-2 r^{2} / w_{0}^{2}\right)$ with $r=\sqrt{x^{2}+y^{2}}$. The incident field with vacuum wave vector $k$ experiences a phase change $\delta \theta=-2 k q(z=$ $0) \equiv-2 k q_{0}$ for the reflected field due to the surface's "piston" motion.

In addition to $\delta \theta$, strain that accompanies surface motion modifies the optical coating and thereby leads to a phase shift $\delta \beta$, with $\delta \beta$ expressed relative to the front surface of the coating. The overall phase shift for the 


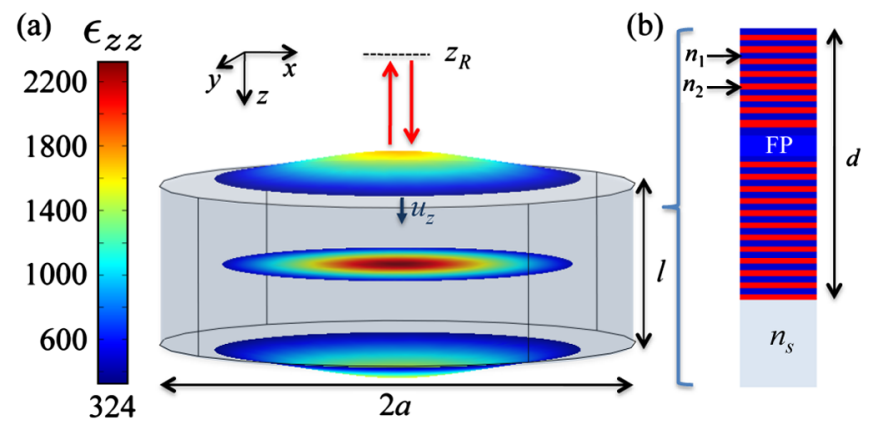

FIG. 1 (color online). (a) The undistorted shape of a substrate of radius $a=1.5 \mathrm{~mm}$ and thickness $l=1 \mathrm{~mm}$ is depicted by the shaded region and wire frame [28]. Axial displacements $u_{z}(x, y, z)$ and strains $\epsilon_{z z}(x, y, z)$ are shown across planes at $z=$ $0, l / 2, l$ (top to bottom) for the eigenmode $\boldsymbol{\xi}_{0}(\boldsymbol{r})$ with eigenfrequency $\omega_{0} / 2 \pi=2.22 \mathrm{MHz}$. Plotted are contours for $u_{z}(x, y, z)$ on which $\epsilon_{z z}(x, y, z)$ is color coded at a time of maximum axial extension, where $u_{z}(x, y, z)=0$ absent excitation. The phase for propagation from $z=z_{R}$ to the substrate and back is modified by surface motion $u_{z}$ at $z=0$. (b) Coating stack for a high reflectivity mirror with embedded FP cavity for high strain sensitivity.

reflected field is then $\delta \Phi=\delta \theta+\delta \beta$, with $|\delta \beta| \ll|\delta \theta|$ for typical optical coatings. We now present new designs for the coating to achieve $\delta \Phi=\delta \theta+\delta \beta \simeq \delta \theta / Q_{0}$.

The coating structure shown in Fig. 1(b) has an embedded resonant layer that gives rise to a rapid phase variation near the cavity resonance [29]. Explicitly, the coating structure is specified as $n_{0}\left(\eta_{1} \eta_{2}\right)^{l} \eta_{1}\left(j \times \eta_{\mathrm{FP}}\right) \times$ $\left(\eta_{1} \eta_{2}\right)^{p-l} n_{s}$. Starting from the vacuum side $n_{0}=1$, there are $l$ double layers $\left(\eta_{1} \eta_{2}\right)^{l}$ with $\eta_{1,2}=\pi / 2$ at the reference wave vector $k_{0}$, followed by an $n_{1}$ layer with $\eta_{1}=$ $\pi / 2$ at $k_{0}$, then the FP $n_{1}$ layer with single-pass phase shift $j \times \eta_{\mathrm{FP}}$ at $k_{0}$, followed by the terminating $p-l$ double layers, and last the substrate with index $n_{s}$. To simplify our discussion, we restrict attention to the case of a "thin" coating with thickness $d \ll a, w_{0}$ and assume that the axial strain in the coating $\epsilon_{z z}^{c}$ equals that at the substrate's surface. See Refs. [22,24] for detailed treatments of multilayer coatings. We stress that axial and transverse strains are explicitly evaluated for the eigenmode $\boldsymbol{\xi}_{0}(\boldsymbol{r})$, including index changes by way of the strain-optic tensor $p_{i j}$. A discussion of thermal noise from the coating itself is deferred to the conclusion.

Results for two particular coatings are given in Fig. 2 for $w_{0} \ll a$. For definiteness, we assume a coating stack made from layers of $\mathrm{SiO}_{2}$ with index $n_{1}=1.45$ and $\mathrm{Ta}_{2} \mathrm{O}_{5}$ with $n_{2}=2.03$. Parts (a),(b) are appropriate to $\boldsymbol{\xi}_{0}(\boldsymbol{r})$ in Fig. 1(a) with coating structure $[p=33, l=8]$. In (a), increased length for the resonant structure leads to greater strain sensitivity, with $|\delta \beta|$ becoming larger relative to $|\delta \theta|$. For $j=16$ there arise "magic" wave vectors $k_{ \pm}$for which $\delta \Phi\left(k_{ \pm}\right)=0$, with the piston phase $\delta \theta$ from surface fluctuations dynamically compensated by the strain-induced coating phase $\delta \beta$. At these "magic wavelengths," the phase of the reflected optical field becomes insensitive to the thermal motion of the surface of the substrate.
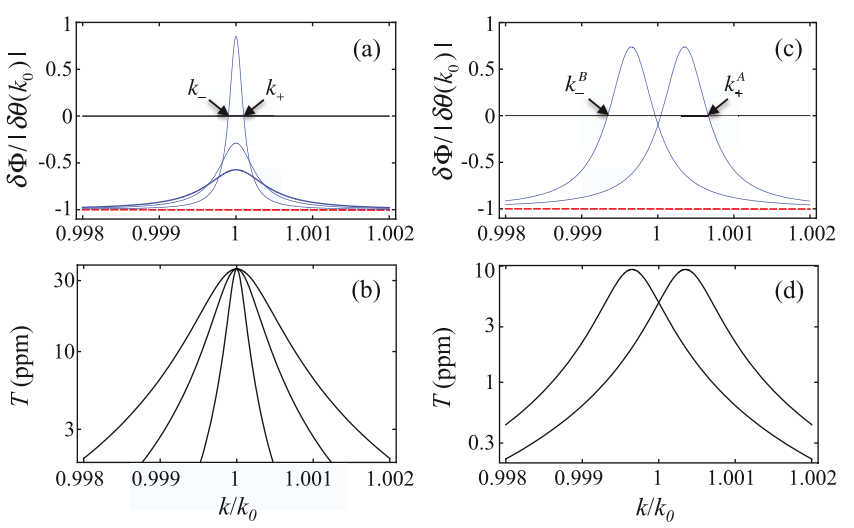

FIG. 2 (color online). Phase shift $\delta \Phi(k)$ and transmission coefficient $T(k)$ for two different coatings illustrated in Fig. 1(b) and specified in the text. (a),(b) The case $\zeta=-1600 / \mathrm{m}$ for increasing phase $j \eta_{\mathrm{FP}}$ of the internal resonant structure. (a) Total phase $\delta \Phi(k)$ with "magic" wave vectors $k_{ \pm}$and (b) associated transmission coefficient $T(k)$ for $j=1,4,16$ with $\eta_{\mathrm{FP}}=\pi$ from widest to narrowest curves. (c),(d) $\delta \Phi(k)$ and $T(k)$ for the case $\zeta=-5000 / \mathrm{m}$ for two coatings with slightly different internal resonances $\eta_{\mathrm{FP}}^{(A, B)}$ with distinct values $k_{-}^{B}, k_{+}^{A}$ for nulling noise from mirrors $B, A$. In all cases, $\delta \Phi(k) /\left|\delta \theta\left(k_{0}\right)\right|$ is plotted for $q_{0}>0$, with $\delta \theta(k)$ given by the dashed lines in (a),(c).

Figures 2(c) and 2(d) investigate the possibility that thermal noise of individual mirrors of an interferometer could be measured in situ. The coating for each of two mirrors $A, B$ is specified by $[p=33, l=7, j=8]$. The thicknesses of the FP layers in the $A, B$ coatings have been adjusted to give $\eta_{\mathrm{FP}}^{(A, B)}=(0.9995,1.0005) \pi$ at $k_{0}$ for the two traces shown. For operation at the lower (upper) value $k_{-}^{B}\left(k_{+}^{A}\right)$ noise from $B(A)$ would be nulled while that from $A(B)$ would be $\sim 80 \%$ of the full piston phase, so that the noise arising from mirrors $A, B$ could be individually measured. For operation at $k / k_{0}=1$, thermal noise from $A$ and $B$ would be suppressed. Panel (d) gives the transmission $T(k)=1-R(k)$ for the two coatings, where $T\left(k_{-}^{B}, k_{+}^{A}\right)$ is consistent with high-finesse measurements. FP resonances away from $k \approx k_{0}$ can also be employed to suppress thermal noise. The process control required for these coatings can be addressed with current technology.

Because the surface strain $|\zeta|$ decreases with increasing size of the substrate, compensation of the piston phase $\delta \theta$ with increasing $a, w_{0}$ requires greater departures from standard coating designs, material specifications, and fabrication procedures. Moreover, not all eigenmodes of oscillation have the proper parity (i.e., $\zeta<0$ ) for compensation by way of the coating designs in Fig. 2 .

A second, more challenging example is thermal noise at frequencies $\omega$ well below the lowest resonance of the mirror substrate. In this quasistatic regime, we must understand the correlation between thermal displacements at depth $z$ and fluctuations of the surface arising from many modes [11]. The foundation for our analysis is the FDT $[7,8]$ as applied by Levin to this setting [13]. 
We consider a substrate in the form of an infinite halfspace with boundary at $z=0$ and extending to $z \geq 0$. Equation (9) in Mindlin [30] provides the Green's function required to deduce the admittance for application of the FDT from Eq. (6.8) of Ref. [8]. Following Ref. [15], we find the spectral correlation for beam-averaged axial displacements $\tilde{q}(z)$ at depths $z_{1}, z_{2}$ to be

$$
\left\langle\tilde{q}\left(z_{2}\right) \tilde{q}\left(z_{1}\right)\right\rangle_{\omega}=\left\langle\tilde{q}_{0} \tilde{q}_{0}\right\rangle_{\omega} N\left(z_{2}, z_{1}\right),
$$

where $\tilde{q}\left(z_{i}\right) \equiv \tilde{q}\left(z_{i}, \omega\right)$ is the Fourier transform of $q\left(z_{i}, t\right)$. $\left\langle\tilde{q}_{0} \tilde{q}_{0}\right\rangle_{\omega}=2 k_{B} T\left(1-\sigma^{2}\right) \phi_{s} / \pi^{3 / 2} w_{0} E \omega$ is the standard result for thermally driven surface displacements, with $E, \sigma$ the Young's modulus and Poisson ratio, respectively, and $\phi_{s}$ the substrate's loss angle. $N\left(z_{2}, z_{1}\right)$ determines the mechanical admittance and is given by

$$
\begin{aligned}
N\left(z_{2}, z_{1}\right)= & \frac{w_{0}}{8 \sqrt{\pi}(1-\sigma)^{2}} \int_{0}^{\infty} d k e^{-k^{2} w_{0}^{2} / 4} f\left(z_{2}, z_{1} ; k\right), \\
f\left(z_{2}, z_{1} ; k\right)= & e^{-k\left|z_{-}\right|\left[3-4 \sigma+k\left|z_{-}\right|\right]+e^{-k z_{+}}[5-12 \sigma} \\
& \left.+8 \sigma^{2}+k(3-4 \sigma) z_{+}+2 k^{2} z_{1} z_{2}\right],
\end{aligned}
$$

with $z_{ \pm}=z_{1} \pm z_{2}$ and $N(z, z)$ plotted in Fig. 3(a). Here, $N(0,0)=1, N\left(z_{1}, z_{2}\right)=N\left(z_{2}, z_{1}\right)[9]$, and $\left\langle\tilde{q}\left(z_{1}\right) \tilde{q}\left(z_{1}\right)\right\rangle_{\omega}$ in Eq. (1) agrees with previous work $[13,15,16]$.

Spectral correlations between thermally driven axial displacements at depths $z_{1}, z_{2}$ are characterized by [31]

$$
C\left(z_{1}, z_{2}\right)=\frac{\left\langle\tilde{q}\left(z_{1}\right) \tilde{q}\left(z_{2}\right)\right\rangle}{\left\langle\tilde{q}\left(z_{1}\right) \tilde{q}\left(z_{1}\right)\right\rangle^{1 / 2}\left\langle\tilde{q}\left(z_{2}\right) \tilde{q}\left(z_{2}\right)\right\rangle^{1 / 2}},
$$

where $C(z, z)=1$ and $\left|C\left(z_{1}, z_{2}\right)\right| \leq 1$. From Eq. (1) and Fig. 3(a), we see that thermal fluctuations of $\tilde{q}(z)$ correlate over length scales set by $w_{0}$, albeit with decreasing amplitude $\langle\tilde{q}(z) \tilde{q}(z)\rangle \propto N(z, z)$ away from the surface.

Figure 3(b) investigates correlation between displacement $\tilde{q}\left(z_{1}\right)$ at $z_{1}$ and axial strain at depth $z_{2}$ by way of the function $Q\left(z_{1} ; z_{2}, \Delta z_{2}\right)=\varepsilon_{z}^{\mathrm{coh}}\left(z_{1}, z_{2}\right) / \varepsilon_{z}^{\mathrm{tot}}\left(z_{2}, \Delta z_{2}\right)$, with $|Q| \leq 1$. Here, $\varepsilon_{z}^{\text {coh }}\left(z_{1}, z_{2}\right) \equiv\left\langle\tilde{q}\left(z_{1}\right)\left[\tilde{q}\left(z_{2}+\Delta z_{2}\right)-\right.\right.$ $\left.\left.\tilde{q}\left(z_{2}\right)\right] / \Delta z_{2}\right\rangle /\left\langle\tilde{q}\left(z_{1}\right)^{2}\right\rangle^{1 / 2}$ is the strain at $z_{2}$ correlated with the displacement $\tilde{q}\left(z_{1}\right)$ at $z_{1}$, while $\varepsilon_{z}^{\text {tot }}\left(z_{2}, \Delta z_{2}\right) \equiv$ $\left\langle\left[\tilde{q}\left(z_{2}+\Delta z_{2}\right)-\tilde{q}\left(z_{2}\right)\right]^{2}\right\rangle^{1 / 2} / \Delta z_{2}$ is the total strain at $z_{2}$.

In Fig. 3(b), the spatial scale for correlation of displacement and strain is again set by $w_{0}$, but now with magnitude reduced by $\sqrt{\Delta z_{2} / w_{0}}$. This scaling of $Q$ arises because the rms strain diverges as $1 / \sqrt{V}$ for thermal fluctuations in a volume $V$ [32], which motivates our use of finite differences to characterize strain. Near the surface with $\Delta z / w_{0} \ll 1$, we find from Eq. (1) that $\varepsilon_{z}^{\text {tot }}(z \simeq 0, \Delta z) \sim\left[\left\langle\tilde{q}_{0} \tilde{q}_{0}\right\rangle /\left(\Delta z w_{0}\right)\right]^{1 / 2}$, where the relevant volume $V \sim \pi w_{0}^{2} \Delta z$. By contrast, the coherent strain $\varepsilon_{z}^{\mathrm{coh}} \equiv \varepsilon_{z}^{\mathrm{coh}}(0,0) \sim-\left\langle\tilde{q}_{0} \tilde{q}_{0}\right\rangle^{1 / 2} / w_{0}$, so that $\left|\varepsilon_{z}^{\mathrm{coh}} / \varepsilon_{z}^{\text {tot }}\right| \propto$ $\sqrt{\Delta z / w_{0}} \ll 1$.

From these exact, 3D results for an elastic half-space, we find that thermally driven surface motion $\tilde{q}_{0}$ arises from substrate strains over distances $z \gtrsim w_{0}$. Hence, an optical coating of thickness $\Delta z=d \ll w_{0}$ employed as a surface-
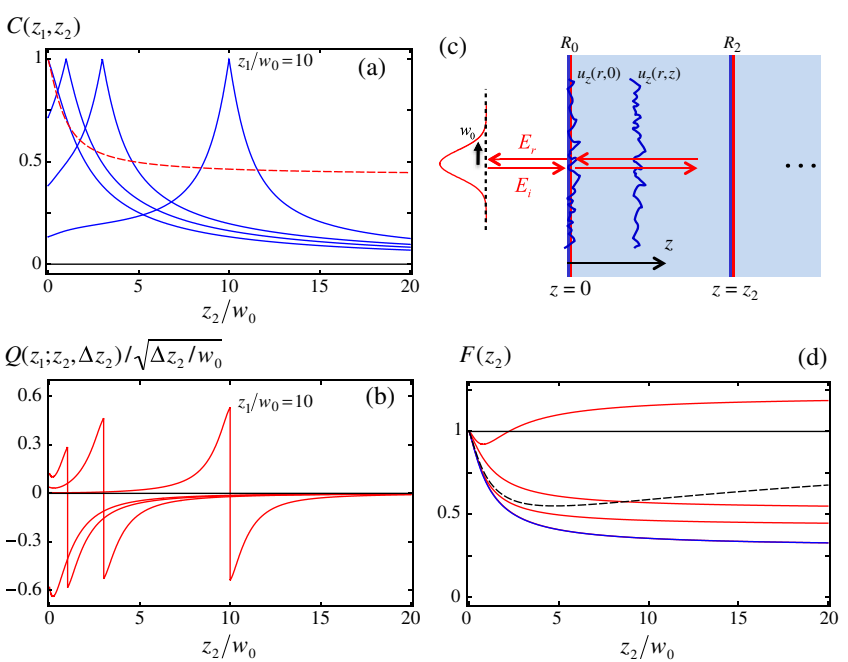

FIG. 3 (color online). (a) Correlation of thermally driven axial displacements $C\left(z_{1}, z_{2}\right)$ and (b) displacement-strain correlation $Q\left(z_{1} ; z_{2}, \Delta z_{2}\right) / \sqrt{\Delta z_{2} / w_{0}}$ versus $z_{2} / w_{0}$ for $z_{1} / w_{0}=0,1,3,10$, with $\Delta z_{2} / w_{0}=10^{-3}$ in (b). The dashed trace in (a) is $N\left(z_{2}, z_{2}\right)$. (c) Illustration of the mirror geometry discussed in the text. (d) Spectral density of phase fluctuations $\boldsymbol{F}\left(z_{2}\right)$ for the reflected field $\boldsymbol{E}_{\boldsymbol{r}}$ in (c) for an $\mathrm{SiO}_{2}$ substrate. From top to bottom, $\alpha=$ $1.5,0.3,1.0,0.7$ with the blue trace for $\alpha=\alpha_{\min }$ overlaying the curve for $\alpha=0.7$. The dashed curve is from a simple model that incorporates incoherent contributions from transverse strains to $\delta \beta$. All curves are for $\sigma=0.2$.

strain sensor as in Fig. 2 will compensate only a small fraction of the phase shift $\delta \theta=-2 k \tilde{q}_{0}$ from the surface motion. Instead, we require a geometry for which $\delta \beta$ has contributions over $z \gtrsim w_{0}$ sufficient to compensate $\delta \theta$.

Figure 3(c) illustrates a mirror geometry that attempts to achieve such compensation. A thin dielectric coating with reflectivity $R_{0}$ is deposited on the substrate's surface, and a second surface is embedded at $z=z_{2} \ll n k w_{0}^{2} / 2$ with reflectivity $R_{2} \rightarrow 1$. In the static case, an incident field $\boldsymbol{E}_{\boldsymbol{i}}$ linearly polarized along $x$ is reflected from this two-mirror geometry to give a field $\boldsymbol{E}_{\boldsymbol{r}} / \boldsymbol{E}_{\boldsymbol{i}}=A e^{i \Gamma(\varphi)}$, where $\varphi$ is the round-trip, internal phase, and $A=1$ for $R_{2}=1$. Thermally driven fluctuations lead to a phase shift $\delta \Phi=$ $-2 k\left[\tilde{q}_{0}+\alpha(k)\left(\tilde{q}\left(z_{2}\right)-\tilde{q}_{0}\right)\right]$ for $\boldsymbol{E}_{\boldsymbol{r}}$, where the piston phase is $\delta \theta=-2 k \tilde{q}_{0}$ and the interferometer phase is $\delta \beta=-2 k \alpha(k)\left(\tilde{q}\left(z_{2}\right)-\tilde{q}_{0}\right) . \alpha(k)$ expresses the sensitivity of the composite cavity $R_{0}, R_{2}$ to strain driven changes in both length and index around $\varphi_{0}$, with

$$
\alpha(k)=n_{s}\left(1-\frac{n_{s}^{2} p_{12}}{2}\right)\left|\frac{d \Gamma\left(\varphi_{0}\right)}{d \varphi}\right|,
$$

where $p_{12}$ is an element of the strain-optic tensor $p_{i j}$ and contributions from fluctuating strains transverse to $z$ are neglected and considered below.

We thus find the spectral density of phase fluctuations for the reflected field $\boldsymbol{E}_{\boldsymbol{r}}$, namely $\left\langle(\delta \Phi)^{2}\right\rangle=\left\langle(\delta \theta)^{2}\right\rangle[(1-$ $\left.\alpha)^{2}+2 \alpha(1-\alpha) N\left(0, z_{2}\right)+\alpha^{2} N\left(z_{2}, z_{2}\right)\right]$. Figure 3(d) plots $\boldsymbol{F}\left(z_{2}\right) \equiv\left\langle(\delta \Phi)^{2}\right\rangle /\left\langle(\delta \theta)^{2}\right\rangle$ for several values of $\alpha$. For fixed $R_{0}$ with $R_{2}=1, \alpha(k)$ varies periodically from 
minimum to maximum over the range $\Delta k=\pi / n_{s} z_{2}$, with a particular $\alpha$ determined by the choice of $k$. $\boldsymbol{F}<1$ represents phase noise reduced below that from thermal fluctuations in the piston phase $\left\langle(\delta \theta)^{2}\right\rangle$. Note that $\alpha=1$ corresponds to direct compensation of the piston phase $\delta \theta \propto \tilde{q}_{0}$ for the reflected field, albeit at the price of noise $\tilde{q}\left(z_{2}\right)$ from fluctuations of the reflecting surface at $z_{2}$. More generally, the minimum $\boldsymbol{F}_{\text {min }}$ for given $z_{2}$ represents a compromise in noise from $\tilde{q}_{0}$ and $\tilde{q}\left(z_{2}\right)$ determined by $\alpha_{\text {min }} \cdot \boldsymbol{F}_{\text {min }} \simeq 0.36$ for $z_{2} \gg w_{0}$ in Fig. 3(d).

An important caveat for Fig. 3(d) is that the full curves omit fluctuations arising from transverse strains $\epsilon_{x x}, \epsilon_{y y}$, which contribute via $p_{i j}$ to $\delta \beta$ and produce a scaling $\boldsymbol{F} \sim$ $z_{2} / w_{0}$ for $z_{2} / w_{0} \gg 1$. A full treatment of these effects is beyond our current analysis. Instead, the dashed curve in Fig. 3(d) is from a simple model based upon the FDT applied to $\epsilon_{x x}, \epsilon_{y y}$ with loss angle $\phi_{s}$ and provides a rough estimate of their incoherent contribution to $\delta \beta$.

The conceptual design in Fig. 3(c) is likely far from optimal. Because the strain field associated with $\psi(r)$ at $z=0$ spreads transversely for $z>0$, it is not well matched to our assumed optical profile with fixed $w(z)=w_{0}$. Geometries with partially reflecting surfaces distributed along $z$ might further reduce $\boldsymbol{F}$. For finite thickness of the substrate, a treatment as in [15] is required, with now the possibility of reflection from the rear surface [27]. More generally, coherent measurements over a range of $k$ values could enhance sensitivity since $\delta \beta(k)$ can be tailored to be distinct from $\delta \theta(k)$.

Although our treatment has been for substrate "Brownian" noise, our methods should be relevant to the suppression of thermal fluctuations from other sources within the substrate $[15,17,18]$. Moreover, variations in the design from Fig. 2 could lead to schemes for suppression of thermal noise within the coating [22]. In contrast to the substrate where strains at $z \ll w_{0}$ have small correlation with $\tilde{q}_{0}$ in the quasistatic regime, coating noise produces surface strains that are highly correlated with $\tilde{q}_{0}$.

Important questions remain about our proposals for noise compensation, including significant fabrication challenges, the additional optical absorption within the coating and substrate, and the need for more complete theoretical analyses. We make no claim of a "magic bullet" for eliminating thermal fluctuations. Rather, our goal is to move beyond a surface-centric view and an incoherent summing of noises to consider instead the coherent character of the underlying stochastic displacements and strains and thereby to suggest new strategies for enhanced sensitivity of optical interferometers.

We gratefully acknowledge the critical insights of $\mathrm{K}$. Thorne, as well as valuable discussions with V. Braginsky, Y. Chen, J. Hall, R. Lalezari, F. Khalili, D. Nelson, and S. Vyatchanin. H. J. K.'s work was made possible by JILA. This research is supported by the NSF and NRC.

Note added.-Subsequent to submission and posting of our work, two manuscripts were posted using coherent effects in optical coatings for reduction of thermal noise $[33,34]$.

*Permanent address: California Institute of Technology MC 12-33, Pasadena, CA 91125, USA.

$\dagger$ Permanent address: Department of Physics, University of Illinois, 1110 West Green Street, Urbana, IL 61801, USA.

[1] V. B. Braginsky and V. P. Mitrofanov, Systems with Small Dissipation (University of Chicago Press, Chicago, 1986).

[2] K. Numata et al., Phys. Rev. Lett. 91, 260602 (2003); 93, 250602 (2004).

[3] A. D. Ludlow et al., Opt. Lett. 32, 641 (2007).

[4] B. C. Barish and R. Weiss, Phys. Today 52, No. 10, 44 (1999).

[5] S. Rowan, J. Hough, and D. R. M. Crooks, Phys. Lett. A 347, 25 (2005).

[6] F. Marquardt, A. A. Clerk, and S.M. Girvin, arXiv:0803.1164.

[7] H. B. Callen and R. F. Greene, Phys. Rev. 86, 702 (1952).

[8] R. F. Greene and H. B. Callen, Phys. Rev. 88, 1387 (1952).

[9] L.D. Landau and E.M. Lifshitz, Statistical Physics (Elsevier, New York, 2006), Chap. XII.

[10] P. R. Saulson, Phys. Rev. D 42, 2437 (1990).

[11] A. Gillespie and F. Raab, Phys. Rev. D 52, 577 (1995).

[12] N. Nakagawa et al., Rev. Sci. Instrum. 68, 3553 (1997).

[13] Yu. Levin, Phys. Rev. D 57, 659 (1998).

[14] F. Bondu, P. Hello, and J.-Y. Vinet, Phys. Lett. A 246, 227 (1998).

[15] Y. Liu and K. Thorne, Phys. Rev. D 62, 122002 (2000).

[16] G. M. Harry et al., Classical Quantum Gravity 19, 897 (2002).

[17] V. B. Braginsky, M. L. Gorodetsky, and S. P. Vyatchanin, Phys. Lett. A 264, 1 (1999).

[18] V. B. Braginsky and S. P. Vyatchanin, Phys. Lett. A 312, 244 (2003).

[19] V. B. Braginsky, M. L. Gorodetsky, and S. P. Vyatchanin, Phys. Lett. A 271, 303 (2000).

[20] E. D. Black et al., Phys. Lett. A 328, 1 (2004).

[21] E. D. Black, A. Villar, and K. G. Libbrecht, Phys. Rev. Lett. 93, 241101 (2004).

[22] G. M. Harry et al., Classical Quantum Gravity 24, 405 (2007).

[23] S. D. Penn et al., Phys. Lett. A 352, 3 (2006).

[24] M. M. Fejer et al., Phys. Rev. D 70, 082003 (2004).

[25] V. B. Braginsky and S. P. Vyatchanin, Phys. Lett. A 324, 345 (2004).

[26] S. Gossler et al., Phys. Rev. A 76, 053810 (2007).

[27] F. Ya. Khalili, Phys. Lett. A 334, 67 (2005).

[28] The parameters for Fig. 1 mimic sapphire, but here for an isotropic medium with Poisson's ratio $\sigma=0.29$, Young's modulus $E=4 \times 10^{11} \mathrm{~Pa}$, and density $\rho=3980 \mathrm{~kg} / \mathrm{m}^{3}$.

[29] H. A. Macleod Thin Film Optical Filters (Taylor \& Francis, Inc., London, 2001), 3rd ed.

[30] R. Mindlin, Physics 7, 195 (1936).

[31] For brevity, henceforth we drop the $\omega$ subscript for \langle\rangle$_{\omega}$.

[32] M. Parrinello and A. Rahman, J. Chem. Phys. 76, 2662 (1982).

[33] M. Evans et al., Phys. Rev. D 78, 102003 (2008).

[34] M. L. Gorodetsky, arXiv:0809.0438. 